\title{
On the simple determination of the self-consistent atomic charges and Madelung potentials in ionic crystals
}

\author{
L.F. Chibotaru ${ }^{\text {a }}$, N.N. Gorinchoi a , A.O. Solonenko ${ }^{\text {a }}$, V.A. Shlyapnikov ${ }^{\text {a }}$, \\ F. Cimpoesu ${ }^{\mathrm{b}}$ \\ a Institute of Chemistry, Academy of Sciences of Moldova, Academiei str. 3, MD2028, Kishinev, Moldavia \\ Institute of Physical Chemistry, Splaiul Independentei 202, Bucharest 77208, Romania
}

Received 9 September 1996; in final form 9 June 1997

\begin{abstract}
A simple method for the self-consistent calculation of the atomic charges and Madelung potentials in ionic crystals is suggested. The method is based on electronegativity equalization and permits the obtaining of good charge distributions. Test calculations are carried out for a number of structures like cubic perovskites, high- $T_{\mathrm{c}}$ copper oxides and mixed valence crystals. (C) 1997 Published by Elsevier Science B.V.
\end{abstract}

Electrostatic or Madelung potentials are important characteristics of ionic and near ionic crystals, determining their electronic structure and physical properties. Numerous calculations of the electronic structure of crystals and molecular fragments of crystals have shown that the Madelung potentials must be included in the calculation schemes to obtain the correct energy level ordering, adequate values of the atomic charges and energy gaps [1-4]. Evaluation of the Madelung potentials implies knowledge of the atomic charges which in their turn can be obtained as the result of complicated quantum chemistry calculations. In this connection the elaboration of simple quantitative methods seems worthwhile. In this Letter a procedure for the self-consistent calculation of atomic charges and Madelung potentials in ionic crystals based on the electronegativity equalization method (EEM) [5] is suggested. It permits the obtaining of good charge distributions and is further simplified for ionic crystals of cubic symmetry.

The electronic energy of an ionic crystal is sup- posed to be a function of atomic charges $q_{k}$ only and is presented in the form

$$
E\left(\ldots, q_{k}, \ldots\right)=\sum_{k=1}^{N} E_{k}\left(q_{k}\right)+E^{M}\left(\ldots, q_{k}, \ldots\right),
$$

where $E_{k}$ is the ionic energy of atom $k\left(E_{k}(0)=0\right)$ and $E^{M}$ is the Madelung energy related to a unit cell; the summation in (1) runs over all the atoms in one unit cell. The functional in Eq. (1) is minimized with respect to all the charge variables under the electroneutrality condition

$\sum_{k} q_{k}=0$

The resulting equations are as follows:

$\frac{\partial E_{k}}{\partial q_{k}}-\varphi_{k}=\lambda, k=1, \ldots, N$.

where the first term on the left-hand side is the charge dependent electronegativity of an isolated atom, $\chi\left(q_{k}\right)$, according to its usual definition [6]; the 
second term is minus the Madelung potential at the position of atom $k$. The two terms together define the electronegativity of atom $k$ in the unit cell. The parameter $\lambda$ is the Lagrange multiplier introduced in order to obey the constraint in Eq. (2). Self-consistent solutions of Eqs. (2) and (3) give some optimized atomic charges, which correspond to a common electronegativity, $\lambda$, for all the atoms in the crystal. The energy expression (1) does not include the covalent contribution, which could be justified only for crystals with preponderant ionic bonding.

Following Jørgensen [7] we approximate the ionic energies by smooth functions of $q_{k}$

$E_{k}\left(q_{k}\right)=\sum_{i=0}^{N} a_{i}^{k} q_{k}^{i+1}$,

making use of known ionization potentials, $I_{n}^{k}$, and affinities $A^{k}$ in the vicinity of the expected value $q_{k}$, and employing the relations:

$I_{n}^{k}=E_{k}(n-1)-E_{k}(n), \quad A^{k}=E_{k}(0)-E_{k}(-1)$.

The atomic electronegativities are obtained from Eq. (1) as follows ${ }^{1}$ :

$\chi_{k}\left(q_{k}\right)=-\sum_{i=0}^{N}(i+1) a_{i}^{k} q_{k}^{i}$.

It is easy to see that this expression is equivalent with Mulliken's definition of the atomic electronegativity $\chi(0)=\left(I_{1}+A\right) / 2$, when the expansion in Eq. (4) is confined to two terms. A generalization of the above approach towards a more detailed account of the charge distribution in the atom will be given elsewhere [8].

The polynomial coefficients in Eqs. (4) and (6) are listed in Table 1 for several atoms. The oxygen atom belongs to a separate case, because it is hardly parameterized in a reliable way from only spectroscopic data, especially in the charge region $-2<q_{\mathrm{o}}$ $<-1$. Additional calculations for some oxygen containing crystals have shown that plausible charges are obtained with the following parameterization:

$\chi_{\mathrm{o}}\left(q_{\mathrm{O}}\right)=-\left(a_{0}^{\mathrm{o}}+a_{1}^{\mathrm{O}} q_{\mathrm{O}}^{2}\right) \exp \left(a_{2}^{\mathrm{o}} q_{\mathrm{O}}^{2}\right)$,

where the coefficients $a_{i}^{o}$ are given in Table 1 .

\footnotetext{
${ }^{1}$ These are equivalent with differential ionization potentials in Jørgensen's treatment [7].
}

Table 1

Parameters for atomic electronegativity expressions (in $\mathrm{eV}$ )

\begin{tabular}{lrrr}
\hline & \multicolumn{1}{c}{$a_{l}^{k}$} & \multicolumn{1}{c}{$a_{2}^{k}$} & $a_{3}^{k}$ \\
\hline $\mathrm{K}$ & -9.39 & 13.73 & - \\
$\mathrm{Cs}$ & -6.71 & 10.60 & - \\
$\mathrm{Ca}$ & 3.23 & 2.88 & - \\
$\mathrm{Sr}$ & 3.02 & 2.67 & - \\
$\mathrm{Ba}$ & 2.81 & 2.39 & - \\
$\mathrm{La}$ & 3.34 & 1.95 & 0.32 \\
$\mathrm{Y}$ & 4.26 & 1.71 & 0.40 \\
$\mathrm{Ti}$ & 5.29 & -0.2 & 1.19 \\
$\mathrm{Zr}$ & 4.88 & 1.36 & 0.59 \\
$\mathrm{Hf}$ & 3.18 & 3.75 & 0.07 \\
$\mathrm{Cu}$ & 2.76 & 4.29 & 0.66 \\
$\mathrm{In}$ & -2.05 & 8.49 & 0.65 \\
$\mathrm{Tl}$ & -2.69 & 9.62 & -0.82 \\
$\mathrm{Sb}$ & 4.96 & 2.71 & 0.46 \\
$\mathrm{Bi}$ & 2.77 & 3.68 & 0.36 \\
$\mathrm{Cl}$ & 11.09 & 6.50 & 0.97 \\
$\mathrm{Br}$ & 10.19 & 5.92 & 0.91 \\
$\mathrm{O}{ }^{\text {a }}$ & 7.54 & 6.95 & 0.0218 \\
\hline
\end{tabular}

${ }^{a}$ Employed in Eq. (7).

The Madelung potentials were calculated within Ewalds' method [9] adapted to a suitable form for further calculations:

$\phi_{k}=\sum_{l} q_{l} F_{k l}$.

The factors $F_{k l}$ are independent of charge and have the meaning of the electrostatic interaction between corresponding sublattices with unit charges when the infinite part of this interaction has been removed:

$$
\begin{aligned}
F_{k l}= & \frac{\pi}{V_{0} G^{2}} \sum_{\vec{g} \neq 0} \cos \left(\vec{g}_{k l}\right) \frac{\exp \left(-g^{2} / 4 G^{2}\right)}{g^{2} / 4 G^{2}} \\
& +\sum_{\vec{n}} \frac{\operatorname{erfc}\left(G\left|\vec{n}+\vec{r}_{k l}\right|\right)}{\left|\vec{n}+\vec{r}_{k l}\right|}-2 \frac{G}{\sqrt{\pi}} \delta\left(\vec{r}_{k l}, 0\right) .
\end{aligned}
$$

Here, $\vec{n}$ and $\vec{g}$ are the direct and inverse lattice vectors, correspondingly, $V_{0}$ is the unit cell volume, $\vec{r}_{k l}$ is the radius vector of the sublattice $k$ against the sublattice $l . G$ is a parameter chosen to accelerate the convergence of sums in (9).

The advantage of this approach is that we need to calculate the lattice sums only once, which is convenient for iterative calculations. In addition, the structural factors obey the relations: $F_{k k}=F_{l l}$ and $F_{k l}=$ $F_{l k}$. It follows from Eqs. (2) and (8) that these 
Table 2

Atomic charges and Madelung potentials for cubic perovskites $\mathrm{ABO}_{3}$

\begin{tabular}{llllllll}
\hline & \multicolumn{2}{l}{ Charges } & & & \multicolumn{3}{c}{ Madelung potentials, eV } \\
\cline { 2 - 3 } \cline { 6 - 8 } & $q_{\mathrm{A}}$ & $q_{\mathrm{B}}$ & $q_{\mathrm{O}}$ & & $\varphi_{\mathrm{A}}$ & $\varphi_{\mathrm{B}}$ & $\varphi_{\mathrm{O}}$ \\
\hline $\mathrm{CaTiO}_{3}$ & 1.76 & 3.46 & -1.74 & -17.81 & -40.41 & 15.87 \\
$\mathrm{SrTiO}_{3}$ & 1.82 & 3.22 & -1.68 & -17.75 & -37.31 & 19.76 \\
$\mathrm{BaTiO}_{3}$ & 1.90 & 3.02 & -1.64 & -17.79 & -34.60 & 18.59 \\
$\mathrm{BaZrO}_{3}$ & 1.92 & 3.57 & -1.83 & -17.55 & -38.24 & 20.12 \\
$\mathrm{BaHfO}_{3}$ & 2.0 & 4.0 & -2.0 & -18.58 & $-\mathbf{4 2 . 6 9}$ & 22.26 \\
\hline
\end{tabular}

Table 3

Charges and Madelung potentials for $\mathrm{La}_{2} \mathrm{CuO}_{4}$

\begin{tabular}{lrrrr}
\hline & \multicolumn{1}{c}{$\mathrm{La}$} & $\mathrm{Cu}$ & \multicolumn{1}{c}{$\mathrm{O}_{\text {axial }}$} & \multicolumn{1}{c}{$\mathrm{O}_{\text {plane }}$} \\
\hline$q$ & 2.87 & 1.94 & -1.89 & -1.95 \\
$\varphi, \mathrm{eV}$ & 26.63 & 27.73 & -19.11 & -20.28 \\
\hline
\end{tabular}

factors are defined with the precision of a constant, which will be chosen in such a way as to have $F_{k k}=0$. Finally, in most structures, there are pairs of sublattices with equivalent vectors $\vec{r}_{k l}$, which allows, in accordance with Eq.(9), further reduction of the number of independent structural factors.

To test the described method, we have calculated the atomic charges and Madelung potentials for the cubic perovskites $\mathrm{ABO}_{3}$. In these lattices the posi- tions of all the atoms correspond to symmetry points, which implies the following dependence of the structural factors on the lattice constant $a$ :

$F_{k l}(a)=A_{k l} / a$.

Here, the parameters $A_{k l}$ play the role of Madelung constants. For the chosen gauge, $F_{k k}=0$, these take the following values:

$A_{\mathrm{AB}}=29.285, A_{\mathrm{BO}}=39.443, A_{\mathrm{AO}}=A_{\mathrm{OO}^{\prime}}=32.442$

where $\mathrm{O}, \mathrm{O}^{\prime}$ denote two of the three oxygen sublattices. The resulting charges and Madelung potentials (Table 2) are in agreement with the results of selfconsistent quantum chemistry calculations [4]. Note the qualitatively correct trend of increasing ionicity in the series $\mathrm{Ca}-\mathrm{Sr}-\mathrm{Ba}$.

Table 4

Charges and Madelung potentials for $\mathrm{YBa}_{2} \mathrm{Cu}_{3} \mathrm{O}_{6}$ and $\mathrm{YBa}_{2} \mathrm{Cu}_{3} \mathrm{O}_{7}$

\begin{tabular}{|c|c|c|c|c|c|c|c|c|}
\hline & $\mathrm{Y}$ & $\mathrm{Ba}$ & $\mathrm{Cu}_{\text {chain }}$ & $\mathrm{Cu}_{\text {plane }}$ & $\mathrm{O}_{\text {axial }}$ & $\mathrm{O}_{\text {plane } x}$ & $\mathrm{O}_{\text {plane } y}$ & $\mathrm{O}_{\text {chain }}$ \\
\hline \multicolumn{9}{|c|}{$\mathrm{YBa}_{2} \mathrm{Cu}_{3} \mathrm{O}_{6}$} \\
\hline$q$ & 2.91 & 1.76 & 0.79 & 1.79 & -1.69 & -1.85 & -1.85 & - \\
\hline$\varphi, \mathrm{eV}$ & 29.42 & 15.60 & 10.52 & 24.26 & -16.67 & -19.47 & -19.47 & - \\
\hline \multicolumn{9}{|c|}{$\mathrm{YBa}_{2} \mathrm{Cu}_{3} \mathrm{O}_{7}$} \\
\hline$q$ & 2.91 & 1.84 & 1.75 & 1.85 & -1.60 & -1.82 & -1.81 & -1.60 \\
\hline$\varphi, \mathrm{eV}$ & 28.36 & 16.82 & 22.44 & 24.04 & -16.34 & -20.17 & -20.04 & -16.34 \\
\hline
\end{tabular}

Table 5

Atomic charges for some halogenostibiates

\begin{tabular}{llllllll}
\hline $\mathrm{A}_{4} \mathrm{M}^{\mathrm{III}} \mathrm{Sb}^{\mathrm{V}} \mathrm{X}_{12}$ & $\mathrm{~A}$ & $\mathrm{M}^{\mathrm{III}}$ & $\mathrm{X}_{\mathrm{eq}}\left(\mathrm{M}^{\mathrm{III}}\right)$ & $\mathrm{X}_{\mathrm{ax}}\left(\mathrm{M}^{\mathrm{III}}\right)$ & $\mathrm{Sb}^{\mathrm{V}}$ & $\mathrm{X}_{\mathrm{eq}}\left(\mathrm{Sb}^{\mathrm{V}}\right)$ & $\mathrm{X}_{\mathrm{ax}}\left(\mathrm{Sb}^{\mathrm{V}}\right)$ \\
\hline$\left(\mathrm{NH}_{4}\right)_{4} \mathrm{Sb}^{\mathrm{III}} \mathrm{Sb}^{\mathrm{V}} \mathrm{Cl}_{12}{ }^{a}$ & 0.882 & 1.867 & -0.573 & -0.588 & 2.307 & -0.663 & -0.671 \\
$\left(\mathrm{NH}_{4}\right)_{4} \mathrm{Sb}^{\mathrm{Ill}} \mathrm{Sb}^{\mathrm{V}} \mathrm{Br}_{12}{ }^{\mathrm{a}}$ & 0.797 & 1.641 & -0.526 & -0.542 & 1.988 & -0.601 & -0.613 \\
$\mathrm{Cs}_{4} \mathrm{Sb}^{\mathrm{III}} \mathrm{Sb}^{\mathrm{V}} \mathrm{Cl}_{12}$ & 0.956 & 1.829 & -0.614 & -0.630 & 2.287 & -0.700 & -0.712 \\
$\mathrm{Cs}_{4} \mathrm{Bi}^{\mathrm{III}} \mathrm{Sb}^{\mathrm{V}} \mathrm{Cl}_{12}$ & 0.947 & 1.735 & -0.603 & -0.620 & 2.212 & -0.683 & -0.674 \\
$\mathrm{Cs}_{4} \mathrm{In}^{\mathrm{III}} \mathrm{Sb}^{\mathrm{V}} \mathrm{Cl}_{12}$ & 0.968 & 1.948 & -0.643 & -0.654 & 2.285 & -0.701 & -0.710 \\
$\mathrm{Cs}_{4} \mathrm{Tl}^{\mathrm{III}} \mathrm{Sb}^{\mathrm{V}} \mathrm{Cl}_{12}$ & 0.967 & 1.589 & -0.587 & -0.590 & 2.275 & -0.689 & -0.706 \\
\hline
\end{tabular}

a In actual calculations the ammonium ion was replaced by the potassium ion. 
As an example of a more complicated crystal structure, high- $T_{\mathrm{c}}$ copper oxides were considered. The calculated atomic charges for $\mathrm{La}_{2} \mathrm{CuO}_{4}$ (Table 3) are close to those obtained by fitting phonon spectra within the point charge model and those obtained by direct quantum chemistry calculations $[10,11]$. As one could expect from the beginning, this compound was found to be more ionic than $\mathrm{Y}-\mathrm{Ba}-$ $\mathrm{Cu}$ superconductors (Table 4). The relatively low value for the charge of $\mathrm{Cu}_{\text {chain }}$ in $\mathrm{YBa}_{2} \mathrm{Cu}_{3} \mathrm{O}_{6}$ as compared with $\mathrm{YBa}_{2} \mathrm{Cu}_{3} \mathrm{O}_{7}$ is due to the absence of oxygen atoms in the chains of the former compound.

Finally, the method was probed to describe the charge distribution in mixed valent crystals. For this purpose some halogenostibiates $\mathrm{A}_{4} \mathrm{M}^{\mathrm{III}} \mathrm{Sb}^{\mathrm{V}} \mathrm{X}_{12}$ were investigated. The crystal lattices in these compounds includes two $\mathrm{K}_{2} \mathrm{PtCl}_{6}$-like unit cells, as a result of the cation alternation. As before, the method describes correctly the change in charges with the nature of the involved atoms. It also accounts for charge disproportionation between stibium ions in $\mathrm{A}_{4} \mathrm{Sb}_{2} \mathrm{X}_{12}$ crystals. Indeed, the disproportionation solution (shown in Table 5) was found to be more stable than the nondisproportionated ones by approximately $0.25 \mathrm{eV}$ for the chloride and $0.12 \mathrm{eV}$ for the bromide compound. The gain in energy comes from the electrostatic interaction of the resulting charge fluctuation on sites. Only the experimentally observed ordering of these fluctuations [12] was considered in the above calculations.

To conclude, the proposed method gives qualitatively correct values of the atomic charges and Madelung potentials for crystals with ionic binding.
One advantage of this method is that it allows the improvement of the Madelung potentials in comparison with the integer charges result, without employing full quantum chemistry calculations. On the other hand, the charges obtained by this method can be used as a starting approximation in self-consistent calculations of the electronic structure of such crystals.

\section{References}

[1] T. Wolfram, R. Hurst, F.J. Morin, Phys. Rev. B 15 (1977) 1151.

[2] T.F. Soules, E.J. Kelley, D.M. Vaught, Phys. Rev. B 6 (1972) 1519.

[3] V.Z. Polinger, N.N. Gorinchoi, I.B. Bersuker, Chem. Phys. 159 (1992) 75.

[4] I.B. Bersuker, N.N. Gorinchoi, T.A. Fedorko, Ferroelectrics 153 (1994) 1.

[5] B.G. Baeke]andt, W.J. Mortier, R.A. Schoonheydt, Struct. Bonding 80 (1993) 187.

[6] R.G. Parr, R.A. Donelly, M. Levy, W.E. Palke, J. Chem. Phys. 68 (1978) 3801, R.P. Iczkowski, J.L. Margrave, J. Am. Chem. Soc. 83 (1961) 3547.

[7] C.K. Jørgensen, Oxidation numbers and oxidation states, Springer-Verlag, Heidelberg, 1969.

[8] F. Cimpoesu, L.F. Chibotaru, I.B. Bersuker and C.I. Lepadatu, Rev. Roumaine Chim. in press.

[9] J.M. Ziman, Principles of the Theory of Solids, University Press, Cambridge, 1972.

[10] G.L. Goodman, D.E. Ellis, E.F. Alp, Soderholm, J. Chem. Phys. 91 (1989) 2983.

[11] G.I. Bersuker, N.N. Gorinchoi, V.Z. Polinger, A.O. Solonenko, Superconductivity 5 (1992) 1005.

[12] K. Prassides, NATO ASI Series C 343 (1991) 201. 